\title{
The Joint Action of Sesquiterpene Lactones from Leaves as an Explanation for the Activity of Cynara cardunculus
}

\author{
Carlos Rial, Benito F. García, Rosa M. Varela, Ascensión Torres, José M. G. Molinillo, \\ and Francisco A. Macías*
} Allelopathy Group, Department of Organic Chemistry, Institute of Biomolecules (INBIO), Campus de Excelencia Internacional
(ceiA3), School of Science, University of Cadiz, C/República Saharaui 7, 11510 Puerto Real, Cadiz, Spain

\begin{abstract}
The work described herein is a continuation of a previous study centered on the bioprospect of cardoon (Cynara cardunculus) leaf extracts through the isolation of secondary metabolites with phytotoxic activity. Chromatographic fractionations of the ethyl acetate extract and spectroscopic analysis showed that the majority of the components were sesquiterpene lactones. Of these compounds, aguerin B, grosheimin, and cynaropicrin were very active on etiolated wheat coleoptile, standard target species, and weed growth. The joint action of binary mixtures of these three active sesquiterpene lactones and one nonactive compound (11,13-dihydroxy-8-desoxygrosheimin) was studied. The activities of fixed-ratio mixtures were assessed on wheat coleoptile. The results can be interpreted with respect to a reference model by considering dose-response analyses and isobolograms with linear regression analyses. A total of 17 binary mixtures at different levels of inhibition $\left(\mathrm{ED}_{25}, \mathrm{ED}_{50}\right.$, and $\left.\mathrm{ED}_{75}\right)$ were studied, and predominantly they responded additively (25). Deviations from additivity included seven synergistic responses and two antagonistic responses. The joint action of major sesquiterpene lactones isolated from $C$. cardunculus can explain the activities observed in extracts and fractions. The results reported here reiterate the utility of the wheat coleoptile bioassay as a quick tool to detect potential synergistic effects in binary mixtures.
\end{abstract}

KEYWORDS: sesquiterpene lactones, cytotoxicity bioassays, binary mixtures, isobole, additive, synergism, antagonist

\section{INTRODUCTION}

In previous work we studied the phytotoxic potential of metabolites from aerial material of Cynara cardunculus (L.), an aggressive perennial invader of grassland communities in Mediterranean climates throughout the world. ${ }^{1}$ However, the invasive actions observed on native plants cannot be explained entirely by their physical characteristics in competition for resources. ${ }^{2}$ Allelopathy could also be involved in the role of the invasive plant C. cardunculus. ${ }^{3-5}$ To know the actual scope of the allelopathic effects, it is important to study not only the pure allelochemicals but also their combined effects. As a consequence, further studies are required in this area.

In a previous study carried out by us on this plant, ${ }^{5}$ the ethyl acetate extract had the highest yield and inhibitory activities on wheat coleoptile and in phytotoxicity bioassays. The ethyl acetate extract showed inhibition values of around $90 \%$ at 0.8 $\mathrm{mg} / \mathrm{mL}$ on wheat coleoptile, with high activity levels retained at lower concentrations $(0.4$ and $0.2 \mathrm{mg} / \mathrm{mL}$ with 87 and $69 \%$ inhibitions, respectively; Figure 1A). The fractions obtained were evaluated in etiolated wheat coleoptile bioassays to biodirect the purification of the allelochemicals (Figure 1B). The most active fractions were D, E, F, and G.

After this bioassay-guided fractionation, the sesquiterpene lactones were isolated and identified as being the major components. ${ }^{5}$ The bioactivity profiles of these compounds were again assayed on wheat coleoptile, with high activities observed-mainly with aguerin B (1), grosheimin (2), and cynaropicrin (3) (Figure 1). ${ }^{5}$

The results outlined above indicate that the bioactivity of the extracts and the fractions can be explained by the presence of the compounds identified, and they highlight the strong potential of C. cardunculus for future use in the biological control of weeds. Comparison of the activity profiles of the extract and the fractions indicates that the activity of the isolated compounds in the fractions obtained from the extracts of this species may be involved in some types of interactions between one another.

Numerous authors agree that allelopathic activity is almost always due to the simultaneous presence of several compounds, as demonstrated by Huang et al. ${ }^{6,7}$ and Einhellig. ${ }^{8}$ Similarly, Rasmussen et al. demonstrated synergistic effects with mixtures of phenolic acids in their studies on grain sorghum germination., 9

Inderjit et al. ${ }^{11}$ found that the joint action of phenolics may depend on the concentration of the compounds in the mixture, and these may act antagonistically at high concentrations and additively at low concentrations.

Studies on binary mixtures are often conducted with the aim of elucidating the effect of one specific chemical on the biological action of another. To achieve this goal, a doseresponse analysis is carried out on various mixture ratios. The projection of the results at specified effect levels, most often the concentration that gives an effect that is half-maximal in grade dose-effect ratios $\left(\mathrm{EC}_{50}\right)$ or a dose that is effective in $50 \%$ of the subjects $\left(\mathrm{ED}_{50}\right)$, on the $x-y$ plane is called an isobole. The results are interpreted statistically using dose-response analyses and isobolograms. ${ }^{12,13}$

Received: June 14, 2016

Revised: July 29, 2016

Accepted: August 3, 2016

Published: August 3, 2016 

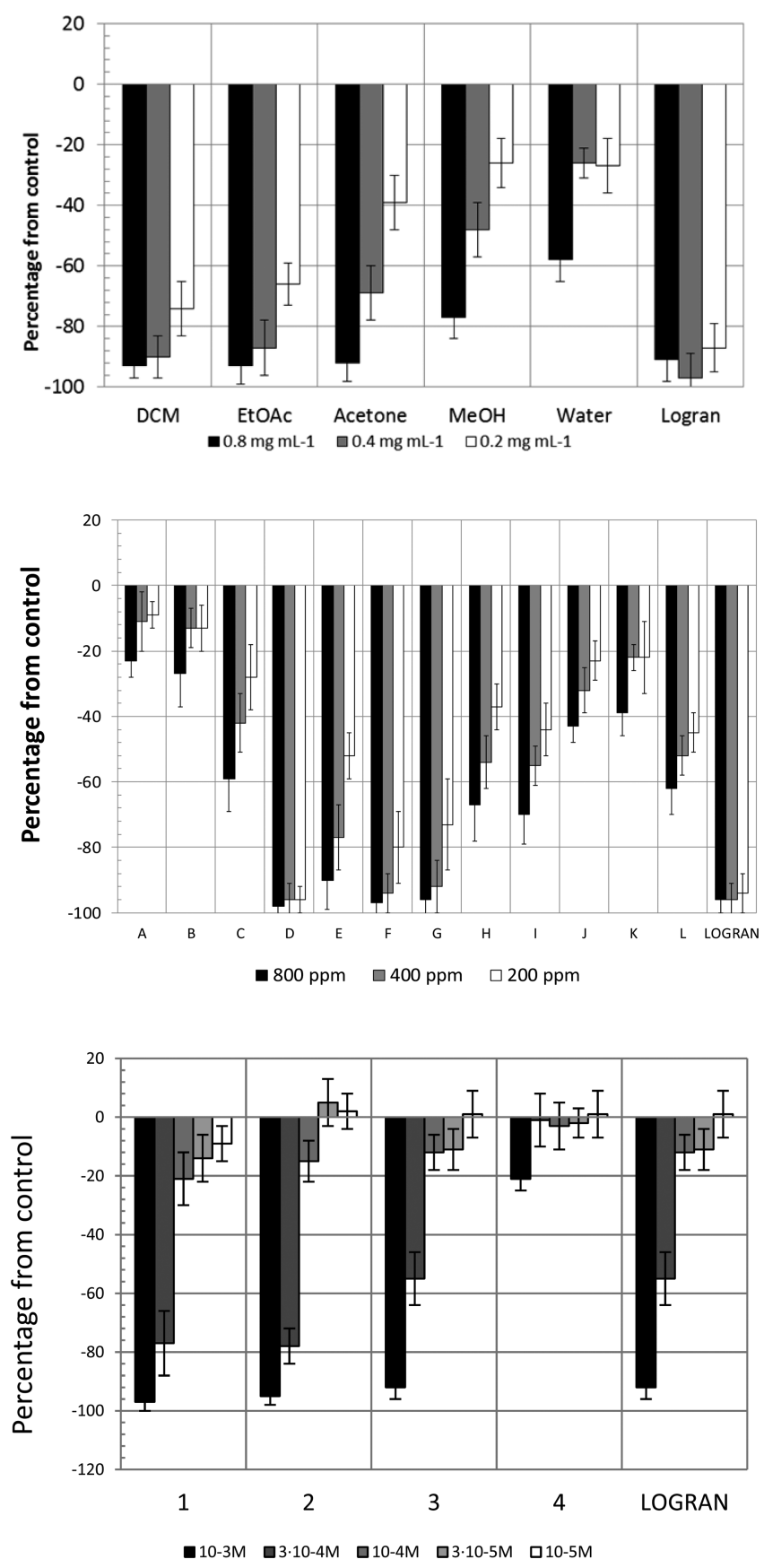

Figure 1. (A, top) Effects of dichloromethane (DCM), ethyl acetate (EtOAc), acetone, methanol $(\mathrm{MeOH})$, and water leaf extracts from cardoon (Cynara cardunculus) and the herbicide Logran on the elongation of etiolated wheat coleoptiles. Values are expressed as percentage difference from control. ${ }^{5}$ (B, middle) Effects of fractions A-L from the ethyl acetate leaf extract of cardoon (C. cardunculus) and the herbicide Logran on the elongation of etiolated wheat coleoptiles. Values are expressed as percentage difference from control. ${ }^{5}$. (C, bottom) Effects of compounds aguerin A (1), grosheimin (2), cynaropicrin (3), and 11,13-dihydroxy-8-desoxygrosheimin (4) from cardoon (C. cardunculus) and the herbicide Logran on the elongation of etiolated wheat coleoptiles.

When two chemicals are evaluated in a specific test system, the shape of the isoboles at different levels of response levels provides a great deal of information on the joint effect. This is useful in the assessment of whether the joint effect of the mixture can be explained with a reference model. To confirm this situation, the cause of the deviation of the reference model should be investigated separately. ${ }^{14-17}$

It is important to design the experiment with the binary mixture (number of doses, replicates, and mixture proportions) and with precision, as all of the aforementioned factors affect the variance of the measured variable and the reproducibility of the shapes of the isoboles.

A complete description of the whole dose-response analysis requires a large number of experiments. Each experiment is costly and laborious and, as a consequence, they are rarely repeated. However, to show the greatest variability in the measured effect, the experiments must be repeated as many times as required.

The aim of the research described here was to evaluate the effects of binary mixtures on the elongation of the etiolated wheat coleoptiles by generating isobolograms and by analyzing the deviations from a reference model, the dependency on the dose level, the effect of combinations of compounds in different proportions, and the isobole asymmetry.

The data employed were obtained from different binary mixtures of the major compounds isolated from cardoon ( $C$. cardunculus): three active compounds, aguerin B (1), grosheimin (2), and cynaropicrin (3), and one inactive compound, 11,13-dihydroxy-8-desoxygrosheimin (4), according to published bioassays. ${ }^{5}$ As outlined above, the performance of mixtures of some compounds has been studied previously; however, the joint action of mixtures of sesquiterpene lactones from invasive plants has not been investigated before. The wheat coleoptile bioassay was employed to detect possible synergistic effects in compounds or mixtures thereof. This test is rapid $(24 \mathrm{~h})^{18}$ and is sensitive to a wide range of bioactive substances such as growth regulators and herbicides, among others. ${ }^{19-21}$

\section{MATERIALS AND METHODS}

Compounds and Binary Mixtures. The following compounds were included in this study: aguerin B (1), grosheimin (2), cynaropicrin (3), and 11,13-dihydroxy-8-desoxygrosheimin (4) (Figure 2). These compounds were isolated previously in our laboratory as the major components of fractions $D-G$ from the

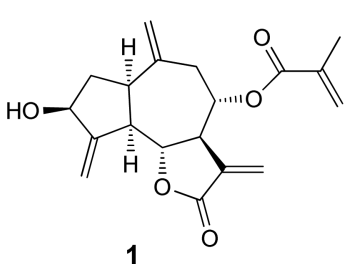

aguerin B

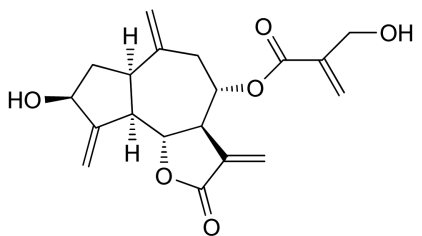

cynaropicrin

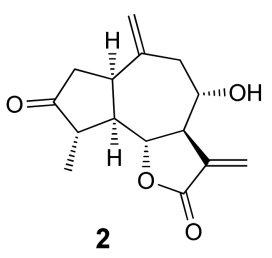

grosheimin

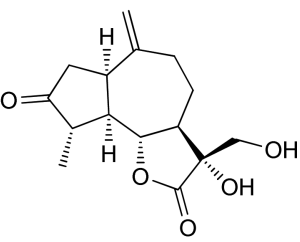

4

11,13-dihydroxy-8desoxygrosheimin
Figure 2. Structures of the major sesquiterpene lactones 1-4 isolated from cardoon (Cynara cardunculus). 
Table 1. Types of Interactions with Student's $t$ Test $(\alpha=0.1)$ and Interaction Index $(\lambda)$ at Different Inhibition Levels $\left(E_{25}\right.$, $\mathrm{ED}_{50}$, and $\left.\mathrm{ED}_{75}\right)$ between the Different Combinations of Binary Mixtures of Isolated Compounds 1-4 from Cardoon (Cynara cardunculus) in the Etiolated Wheat Coleoptile Bioassay ${ }^{a}$

\begin{tabular}{|c|c|c|c|c|c|c|c|c|c|c|c|c|}
\hline \multirow[b]{3}{*}{ combination ratio } & \multicolumn{12}{|c|}{ binary mixture of compounds } \\
\hline & \multicolumn{2}{|c|}{$1 / 2$} & \multicolumn{2}{|c|}{$1 / 3$} & \multicolumn{2}{|c|}{$1 / 4$} & \multicolumn{2}{|c|}{$2 / 4$} & \multicolumn{2}{|l|}{$3 / 2$} & \multicolumn{2}{|c|}{$3 / 4$} \\
\hline & $\mathrm{ED}_{25}$ & $\lambda$ & $\mathrm{ED}_{25}$ & $\lambda$ & $\mathrm{ED}_{25}$ & $\lambda$ & $\mathrm{ED}_{25}$ & $\lambda$ & $\mathrm{ED}_{25}$ & $\lambda$ & $\mathrm{ED}_{25}$ & $\lambda$ \\
\hline $3: 1$ & Add & 1.02 & Add & 1.35 & & & Add & 1.09 & Ant* & 1.74 & Ant* & 5.16 \\
\hline $3: 2$ & & & & & Add & 1.14 & & & & & & \\
\hline $1: 1$ & Add & 1.25 & Add & 0.87 & & & \# & \# & Add & 1.22 & \# & \# \\
\hline $2: 3$ & & & & & Add & 1.12 & & & & & & \\
\hline \multirow[t]{3}{*}{$1: 3$} & Add & 0.89 & Add & 0.86 & & & \# & \# & \# & \# & $\#$ & $\#$ \\
\hline & \multicolumn{12}{|c|}{ binary mixture of compounds } \\
\hline & \multicolumn{2}{|c|}{$1 / 2$} & \multicolumn{2}{|c|}{$1 / 3$} & \multicolumn{2}{|c|}{$1 / 4$} & \multicolumn{2}{|c|}{$2 / 4$} & \multicolumn{2}{|c|}{$3 / 2$} & \multicolumn{2}{|c|}{$3 / 4$} \\
\hline combinatio ratio & $\mathrm{ED}_{50}$ & $\lambda$ & $\mathrm{ED}_{50}$ & $\lambda$ & $\mathrm{ED}_{50}$ & $\lambda$ & $\mathrm{ED}_{50}$ & $\lambda$ & $\mathrm{ED}_{50}$ & $\lambda$ & $\mathrm{ED}_{50}$ & $\lambda$ \\
\hline $3: 1$ & Add & 0.76 & Add & 1.46 & & & Add & 1.18 & Syn* & 0.70 & & \\
\hline $3: 2$ & & & & & Add & 0.71 & & & & & & \\
\hline $1: 1$ & Add & 0.85 & Add & 0.76 & & & \# & $\#$ & Add & 0.93 & & \\
\hline $2: 3$ & & & & & Add & 0.85 & & & & & & \\
\hline \multirow[t]{3}{*}{$1: 3$} & Syn* & 0.67 & Add & 0.84 & & & $\#$ & \# & \# & \# & & \\
\hline & \multicolumn{12}{|c|}{ binary mixture of compounds } \\
\hline & \multicolumn{2}{|c|}{$1 / 2$} & \multicolumn{2}{|c|}{$1 / 3$} & \multicolumn{2}{|c|}{$1 / 4$} & \multicolumn{2}{|c|}{$2 / 4$} & \multicolumn{2}{|r|}{$3 / 2$} & \multicolumn{2}{|c|}{$3 / 4$} \\
\hline combination ratio & $\mathrm{ED}_{75}$ & $\lambda$ & $\mathrm{ED}_{75}$ & $\lambda$ & $\mathrm{ED}_{75}$ & $\lambda$ & $\mathrm{ED}_{75}$ & $\lambda$ & $\mathrm{ED}_{75}$ & $\lambda$ & $\mathrm{ED}_{75}$ & $\lambda$ \\
\hline $3: 1$ & Syn* & 0.57 & Add & 1.60 & & & Add & 1.27 & Syn* & 0.35 & & \\
\hline $3: 2$ & & & & & Syn* & 0.44 & & & & & & \\
\hline $1: 1$ & Syn* & 0.59 & Add & 0.69 & & & $\#$ & \# & Add & 0.84 & & \\
\hline $2: 3$ & & & & & Add & 0.65 & & & & & & \\
\hline $1: 3$ & Syn* & 0.54 & Add & 0.84 & & & $\#$ & \# & \# & $\#$ & & \\
\hline
\end{tabular}

${ }^{a}$ Add, additive; Syn, synergistic; Ant, antagonistic; *, Student's $t$ test $(\alpha=0.1)$; \#, slope is not significantly different from 0 "ANOVA in linear regression".

Table 2. Comparison of the Additive and Experimental Regression Lines for Binary Mixtures of Compounds with Student's $t$ Test Test for Parallelism $(\alpha=0.05)$ (If $t_{\text {calcd }}>t$ There Is a Significant Difference)

\begin{tabular}{|c|c|c|c|c|c|c|c|c|c|c|}
\hline \multirow[b]{3}{*}{ combination ratio } & \multicolumn{10}{|c|}{ binary mixture of compounds } \\
\hline & \multicolumn{2}{|c|}{$1 / 2$} & \multicolumn{2}{|c|}{$1 / 3$} & \multicolumn{2}{|c|}{$1 / 4$} & \multicolumn{2}{|c|}{$2 / 4$} & \multicolumn{2}{|c|}{$3 / 2$} \\
\hline & $t_{\text {calcd }}$ & $t$ & $t_{\text {calcd }}$ & $t$ & $t_{\text {calcd }}$ & $t$ & $t_{\text {calcd }}$ & $t$ & $t_{\text {calcd }}$ & $t$ \\
\hline 3:1 & 2.31 & 2.20 & 0.42 & 2.20 & & & 0.45 & 2.45 & 2.67 & 2.20 \\
\hline $3: 2$ & & & & & 4.32 & 2.57 & & & & \\
\hline $1: 1$ & 3.30 & 2.20 & 0.91 & 2.20 & & & & & 2.20 & 2.20 \\
\hline $2: 3$ & & & & & 0.93 & 2.45 & & & & \\
\hline $1: 3$ & 2.57 & 2.20 & 0.12 & 2.20 & & & & & & \\
\hline
\end{tabular}

ethyl acetate extract of aerial plant material of cardoon (C. cardunculus), which was collected in Jerez de la Frontera (Cadiz, Spain) in the fifth growth stage. ${ }^{5}$ Compounds $\mathbf{1 - 4}$ were obtained from fractions $\mathrm{D}-\mathrm{G}$ as follows: fraction D yielded compounds $\mathbf{1}(23 \mathrm{mg})$ and $\mathbf{2}(7.1 \mathrm{mg})$; fraction E, compound 2 (10 mg); fraction F, compound 3 (241 mg); and fraction $\mathrm{G}$, compound $4(36 \mathrm{mg})$.

In the combined action studies on these sesquiterpene lactones, two and three ratios were examined for each binary mixture, respectively. The ratios of the compounds in the mixtures varied depending on the amounts of the compounds available (Table 1).

Coleoptile Bioassay. Wheat seeds (Triticum aestivum L. cv. Catergo) were placed in Petri dishes, and the tests were carried out according to the guidelines published by Hancock ${ }^{22}$ and Nistch. ${ }^{23}$ Compounds and binary mixtures were predissolved in DMSO $(0.1 \%)$ and diluted in phosphate-citrate buffer containing $2 \%$ sucrose at $\mathrm{pH}$ 5.6 to the final bioassay concentrations $(1.0,0.3,0.1,0.03$, and 0.01 $\mathrm{mM}$ for individual compounds; $0.3,0.1,0.09,0.05$, and $0.03 \mathrm{mM}$ for binary mixtures of active compounds; and $0.3,0.1,0.06,0.03$, and 0.02 $\mathrm{mM}$ for binary mixtures of active and inactive compounds, with different proportions: $3: 1,3: 2,1: 1,2: 3$, and 1:3).
The commercial herbicide Logran was used as an internal reference, and the concentrations and the conditions of bioassay were the same as reported previously. ${ }^{18,24}$ In the reproducibility of the dose-response curve (in the bioassay of the binary mixtures), pure compound was used as control.

The coleoptiles were measured by digitalization of their images, and data were statistically analyzed using Welch's test. ${ }^{25}$

Isobolographic Data Analysis. The dose-effect curves were constructed from data obtained in the coleoptile bioassay, and they represent the effect against the logarithm of the dose. The logarithmic transformation results in an S-shaped curve that is approximately linear in the midrange. ANOVA in linear regression was applied to determine whether the slope was significantly different from 0 with $95 \%$ confidence limits (for $\log$ (dose)-effect data this is a test to determine whether the effect is dose dependent). ${ }^{26}$

The interactions between the tested compounds were characterized by performing an isobolographic analysis. Isobolographs were based on $\mathrm{ED}_{25}-\mathrm{ED}_{75}$ values (doses that produce $25-75 \%$ inhibition with respect to the control) obtained when the compound was administered alone or in combination. According to the method 

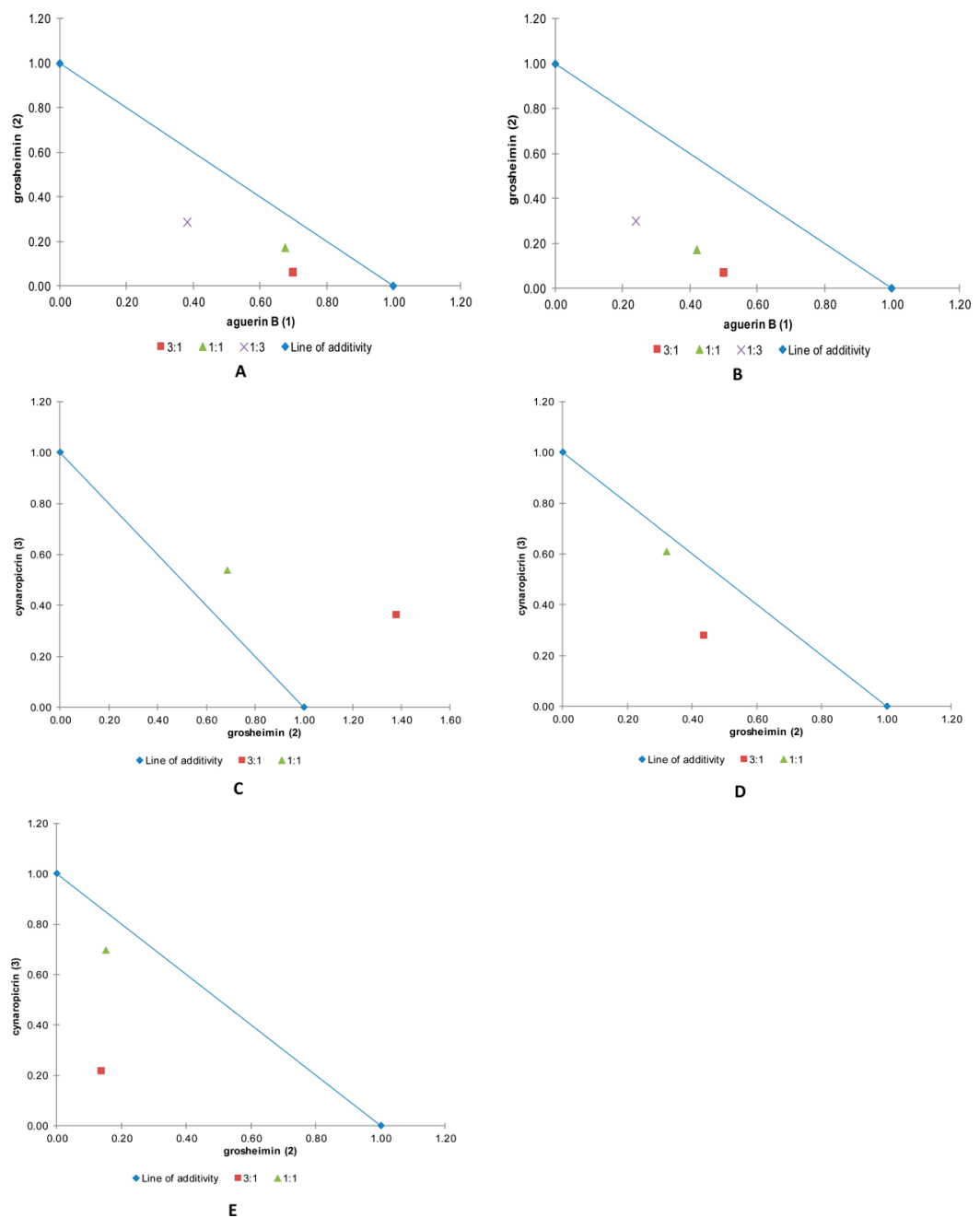

Figure 3. Normalized isobolograms for the binary mixtures with aguerin B (1), grosheimin (2), and cynaropicrin (3) in the etiolated wheat coleoptile bioassay: (A) binary mixture $1 / 2$ at $\mathrm{ED}_{50}$; (B) binary mixture $\mathbf{1 / 2}$ at $\mathrm{ED}_{75}$; (C) binary mixture $\mathbf{3 / 2}$ at $\mathrm{ED}_{25}$; (D) binary mixture $\mathbf{3 / 2}$ at $\mathrm{ED}_{50}$; $(\mathrm{E})$ binary mixture $3 / 2$ at $\mathrm{ED}_{75}$. The axes represent the dose of each compound for the measured effect in values in per unit.

used by Tallarida, ${ }^{26}$ the theoretical additive doses $\left(Z_{\text {add }}\right)$ with their variance $\left(V_{\text {add }}\right)$ for each combination were computed from the equieffective doses $\left(\mathrm{ED}_{25}-\mathrm{ED}_{75}\right)$ of the single compounds. The experimental data $\left(Z_{\text {expt }}\right)$ and their variance $\left(V_{\text {exptl }}\right)$ were determined from the respective dose-response curves. A statistical comparison was made by applying Student's $t$ test with $90 \%$ confidence limits, between $Z_{\text {exptl }}$ (experimental values) and $Z_{\text {add }}$ (theoretical values). ${ }^{26}$

The level of synergism was estimated by considering the values of the interaction index $(\lambda)$. These values indicate the proportion of the $\mathrm{ED}_{25}, \mathrm{ED}_{50}$, and $\mathrm{ED}_{75}$ values of the individual compound that accounts for the corresponding $\mathrm{ED}_{25}, \mathrm{ED}_{50}$, and $\mathrm{ED}_{75}$ values of the combination. An additive interaction corresponds to values close to 1 , an antagonistic interaction is indicated by values $>1$, and a synergistic interaction is associated with values of $<1$. The interaction index is given by $\lambda=Z_{\text {explt }} / Z_{\text {add. }}{ }^{27}$

Data Analysis: Comparison of the Additive and Experimental Regression Lines. The dose-effect data for the compounds, for each fixed ratio, were used to construct a theoretical additive curve according to the method described by Tallarida. ${ }^{28}$ Comparison of the linear regressions of the additive and experimental curves represents a test for parallelism, where the hypothesis to be tested is the difference between slope, and the test uses the $t$ distribution, which is obtained by Student's $t$ test with $95 \%$ confidence limits (Table 2). ${ }^{26,28}$

\section{RESULTS AND DISCUSSION}

Isobolographic Analysis of Chemical Interactions in the Coleoptile Bioassay. The results of the studies on binary mixtures in bioactivity assays can be interpreted in relation to reference models by the use of isobolograms in conjunction with the "Independent Similar Action" model, as described by Bliss. ${ }^{28}$ When the mixtures include chemicals that synergize or antagonize the effects of other components, this approach may be become more difficult. ${ }^{17}$

The isobole method is independent of the mechanism of action and applies under most conditions. Assumptions are not made with respect to the behavior of each agent, and the method is therefore applicable to multiple component mixtures. An isobologram is an "iso-effect" graph in which the axes are the doses of the individual compounds $\left(D_{a}, D_{\mathrm{b}}\right)$ and dose combinations $\left(d_{a}, d_{\mathfrak{b}}\right)$ that cause the same effect. If the compounds do not interact, the isobole is a straight line (linear additivity or zero interaction). ${ }^{29}$ However, if synergism occurs, the required dose of the combination to produce the same effect will be less than the sum of the individual doses, and as shown this lies below the line of additivity in the isobologram. If the interaction shows antagonism, the required dose of the combination is greater than expected, and these points are located above the line of additivity. ${ }^{18}$ Student's $t$ tests were 

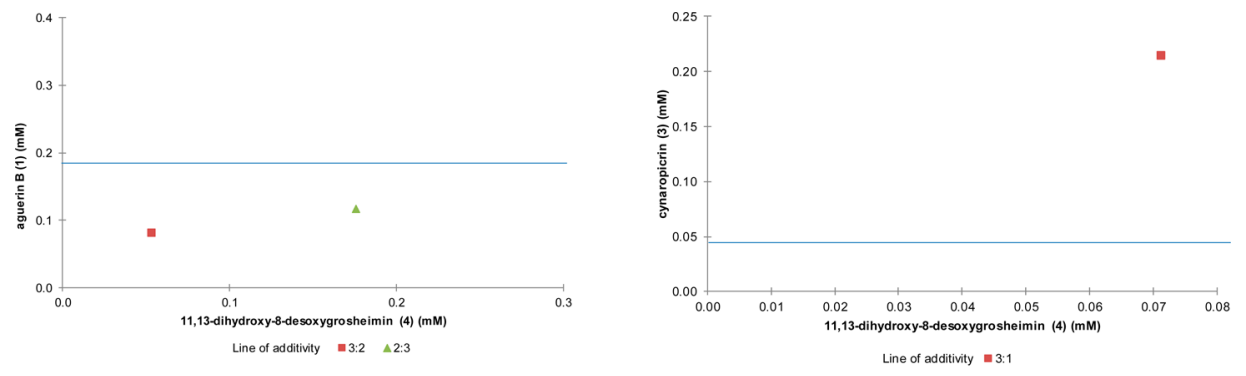

A

B

Figure 4. Isobolograms for the binary mixtures with the inactive compound 11,13-dihydroxy-8-desoxygrosheimn (4) with aguerin B (1) and cynaropicrin (3) in the etiolated wheat coleoptile bioassay: (A) binary mixture $1 / 4$ at $\mathrm{ED}_{75}$; (B) binary mixture $3 / 4$ at $\mathrm{ED}_{25}$.

Table 3. Comparison of Theoretical and Experimental Doses in Binary Mixtures from Aguerin B (1) and Grosheimin (2) at $\mathrm{ED}_{75}$

\begin{tabular}{|c|c|c|c|c|c|c|}
\hline \multirow[b]{2}{*}{ combination ratio } & \multicolumn{2}{|c|}{ theor dose $(M)$ (at inhibition $75 \%$ ) } & \multicolumn{2}{|c|}{ exptl dose $(\mathrm{M})$ (isobologram-ED $\mathrm{D}_{75}$ ) } & \multicolumn{2}{|c|}{ reduction dose $(\%)$} \\
\hline & aguerin $B(1)$ & grosheimin (2) & aguerin $\mathrm{B}(1)$ & grosheimin (2) & aguerin $B(1)$ & grosheimin (2) \\
\hline $3: 1$ & $1.81 \times 10^{-4}$ & $6.05 \times 10^{-5}$ & $1.04 \times 10^{-4}$ & $3.46 \times 10^{-5}$ & 42.54 & 42.81 \\
\hline $1: 1$ & $1.46 \times 10^{-4}$ & $1.45 \times 10^{-4}$ & $8.68 \times 10^{-5}$ & $8.63 \times 10^{-5}$ & 40.55 & 40.48 \\
\hline $1: 3$ & $9.21 \times 10^{-5}$ & $2.74 \times 10^{-4}$ & $4.98 \times 10^{-5}$ & $1.48 \times 10^{-4}$ & 45.93 & 45.99 \\
\hline
\end{tabular}

carried out in the isobolographic analysis to evaluate the interactions between the compounds. ${ }^{29}$ The magnitude or intensity of the effect was evaluated from the values of the interaction index, $\lambda(\lambda \sim 1$ additive interaction, $\lambda>1$ antagonistic interaction, and $\lambda<1$ synergistic interaction). ${ }^{18}$

The interactions of 17 binary mixtures with the sesquiterpene lactones aguerin B (1), grosheimin (2), cynaropicrin (3), and 11,13-dihydroxy-8-desoxygrosheimin (4), isolated from $C$. cardunculus, were evaluated in the etiolated wheat coleoptile bioassay. Student's $t$ test was applied with a confidence level of $90 \%$, and 25 additive interactions, 7 synergistic interactions, and 2 antagonistic interactions were detected at different levels of inhibition $\left(\mathrm{ED}_{25}, \mathrm{ED}_{50}\right.$, and $\left.\mathrm{ED}_{75}\right)$, as shown in Table 1 .

Of all the combinations of binary mixtures tested from the four sesquiterpene lactones $\mathbf{1 - 4}$ in the etiolated wheat bioassay, it is noteworthy that all four compounds were involved in all seven synergistic effects. Even compound 4, which showed lower activity than the other three compounds $(1-3)$, as found in our previous study, ${ }^{5}$ was involved in these effects. The most noteworthy isobolograms of binary mixtures of these compounds in different proportions are shown in Figures 3 and 4 . In these graphs, the axes in the normalized isobologram represent the dose of each compound for the measured effect in values in per unit.

It can be seen from the normalized isobologram $\left(\mathrm{ED}_{50}\right)$ that for the binary mixture of aguerin B (1)/grosheimin (2) a synergistic effect is obtained when the two compounds are in a $1: 3$ ratio (Figure $3 \mathrm{~A}$ ). This ratio (1:3) was found to be significantly different on applying Student's $t$ test, thus providing evidence of a synergistic effect. In contrast, when compound 1 was present in a higher proportion $(3: 1)$ or at the same level (1:1), an additive effect was observed (Figure 3A). It can be seen from the figure that these ratios are below the line of additivity, and significant differences were not found on applying Student's $t$ test, thus indicating that an additive effect was obtained. The application of a normalized isobologram $\mathrm{ED}_{25}$ for these binary mixtures $(\mathbf{1} / \mathbf{2})$ showed that at low doses there were additive effects for the three ratios tested (3:1, 1:1, and 1:3) (Table 1). The normalized isobologram $\mathrm{ED}_{75}$ showed synergistic effects for all ratios tested (3:1, 1:1, and 1:3) (Figure
$3 \mathrm{~B})$. In the normalized isobologram for $\mathrm{ED}_{75}$, all of the ratios were located below the line of additivity and significant differences were observed on applying Student's $t$ test, thus showing a synergistic effect. Furthermore, for $\mathrm{ED}_{75}$ a synergistic effect for the combination of these two compounds (1 and 2) can be further illustrated by the results in Table 3. The theoretical concentration values, taking into account the additive effect in this case, and those obtained experimentally are shown in Table 3. Thus, the theoretical values are those for the pure compounds obtained in the bioassay-guided isolation, which showed $75 \%$ inhibition at $3.10^{-4} \mathrm{M}$ (Figure 1C), and those obtained experimentally are from the combinations in the normalized $\mathrm{ED}_{75}$ isobolograms. It can be seen that fewer doses of each compound are required in the respective mixtures (between 40.48 and $45.99 \%$ lower values) than the values calculated theoretically for an additive effect that caused this $75 \%$ inhibition (Table 3 ).

The results for the types of interactions in the normalized isobolograms $\left(\mathrm{ED}_{25}, \mathrm{ED}_{50}\right.$, and $\left.\mathrm{ED}_{75}\right)$ were therefore not constant for the different ranges of the measured effects. It is possible to consider that these results possibly indicate a change in the type of interaction depending on the doses. For example, for the binary mixture of compounds 1 and 2 in a 1:3 ratio, the use of isobolograms at different levels indicates that additivity occurs at low doses $\left(\mathrm{ED}_{25}\right)$; at the doses that cause 50 and $75 \%$ inhibition, the effect of synergism was identified, with synergism at $\mathrm{ED}_{75}$ being more pronounced than that at $\mathrm{ED}_{50}$, because the interaction index is lower $(\lambda=0.54)$ (Table 1$)$. Furthermore, at the highest level tested $(75 \%)$ an even stronger synergistic interaction was found when compared to the other ratios measured (3:1 and 1:1).

The results for the normalized isobologram $\left(\mathrm{ED}_{25}, \mathrm{ED}_{50}\right.$, and $\mathrm{ED}_{75}$ ) for aguerin $\mathrm{B}(1)$ /cynaropicrin (3) binary mixtures in different proportions are shown in Table 1 . The results are consistent with an additive effect when the two compounds are present in 3:1, 1:1, and 1:3 ratios (Table 1).

The binary mixture aguerin B (1)/11,13-dihydroxy-8desoxygrosheimin (4) showed an additive effect for the two ratios tested (3:2 and 2:3). The corresponding results $\left(\mathrm{ED}_{25}\right.$ and $\left.\mathrm{ED}_{50}\right)$ are shown in Table 1 . However, when the binary 
mixture $1 / 4$ reaches $75 \%$ inhibition, a synergistic effect is observed for the 3:2 ratio, as shown in the isobologram $\left(\mathrm{ED}_{75}\right)$ (Figure 4A). In the graph both ratios are located below the line of additivity, but only for the 3:2 ratio was a significant difference observed on applying Student's $t$ test, thus showing a synergistic effect. This binary mixture differs from the others because it is composed of an active sesquiterpene lactone (1) and a nonactive compound $(4) ;^{5}$ however, synergism could be detected between these compounds under the experimental conditions. Similarly, nonactive compound $\mathbf{4}$ was also combined with two other active compounds, grosheimin (2) and cynaropicrin (3). In the binary mixture grosheimin (2)/ 11,13-dihydroxy-8-desoxygrosheimin (4), the isobolograms $\left(\mathrm{ED}_{25}, \mathrm{ED}_{50}\right.$, and $\left.\mathrm{ED}_{75}\right)$ show only an additive effect for the $3: 1$ ratio (Table 1). In combination with the other active lactone (3), the binary mixture cynaropicrin (3)/11,13dihydroxy-8-desoxygrosheimin (4) showed an antagonistic effect at a level of $25 \%$ inhibition for the $3: 1$ ratio, as shown in the isobologram (Figure 4B). In this isobologram the combination ratio $(3: 1)$ is located above the line of additivity, and a significant difference was observed on applying Student's $t$ test, which indicates an antagonistic effect. Thus, the results obtained on using the inactive compound 4 in binary mixtures with active compounds 1, 2, and 3 showed three types of interactions, antagonism with compound 3, additivity with compounds 1 and 2, and synergism with compound $\mathbf{1}$, under the conditions tested, as well as synergistic and antagonistic effects; compound 4 is present at a lower level than compounds 1 and 3 in the binary mixtures tested (3:2 and $3: 1$, respectively). This is not the first such case to be reported and, as Berenbaum explained in his paper, ${ }^{29}$ it is possible that one type of synergy, that is, potentiation, occurs when a compound does not show activity in its own right but enhances the activity of another active compound. In some circumstances, unequivocal decisions can be made with even less information about the dose-response curve, because if one of the compounds does not produce the effect of the combination at any dose and the effect of the combination differs from that of the active compound, then some sort of interaction must be present.

Similar results were obtained by Junio and co-workers, who argued that three flavonoids isolated from Hydrastic canadensis synergistically enhance the antimicrobial activity of the alkaloid berberine (also a constituent of $H$. canadensis) against Staphylococus aureus. These flavonoids do not have inherent antimicrobial activity against $S$. aureus. ${ }^{30}$

For the binary mixture of cynaropicrin (3)/grosheimin (2), the interactions in the normalized isobolograms $\left(\mathrm{ED}_{25}, \mathrm{ED}_{50}\right.$, and $\left.E D_{75}\right)$ were different depending on the ranges of effects measured. This indicates that the type of interaction could change according to the doses. Antagonism is detected in the normalized isobologram $\mathrm{ED}_{25}$ for the 3:1 ratio, whereas an additive effect is observed for the $1: 1$ ratio (Figure $3 \mathrm{C}$ ). In this case both ratios are located above the line of additivity, but only the 3:1 ratio showed a significant difference on applying Student's $t$ test, and this indicated an antagonist effect. On the other hand, in the normalized isobolograms $\mathrm{ED}_{50}$ (Figure 3D) and $\mathrm{ED}_{75}$ (Figure $3 \mathrm{E}$ ) synergism and additivity were observed for the 3:1 and 1:1 ratios, respectively. In both graphs, the 3:1 and $1: 1$ ratios are located below the line of additivity, but only for the 3:1 ratio was a significant difference observed on applying Student's $t$ test, which showed a synergistic interaction. Isobolograms were constructed at different levels for the binary mixture of compounds $3 / 2$ in a $3: 1$ ratio, and it was found that antagonism occurs at low doses. Synergistic effects were identified for the doses that caused medium (50\%) and high (75\%) inhibition. Furthermore, the synergistic interaction at $\mathrm{ED}_{75}$ is more pronounced than that at $\mathrm{ED}_{50}$ by virtue of the lower interaction index $(\lambda=0.35)$.

In summary, the results obtained by studying the interactions in binary mixtures of the four sesquiterpene lactones (1-4) led to a total of 17 binary mixtures at different levels of inhibition $\left(\mathrm{ED}_{25}, \mathrm{ED}_{50}\right.$, and $\left.\mathrm{ED}_{75}\right)$, most of which showed additive effects (25) followed by synergistic effects (7), and, to a lesser extent, antagonism (2). Therefore, of all the possible interactions, the additive and synergistic effects were more prevalent than the antagonistic ones. These results are consistent with the findings reported by Caccioni in a holistic approach ${ }^{31}$ and allow us to explain the activity profile of the ethyl acetate extract in the etiolated wheat coleoptile bioassay, which is possibly due to the prevalence of more additive and synergistic effects rather than antagonist effects.

Combination Analysis: Comparison of the Additive and Experimental Regression Lines. The identification of sets of equally effective dose combinations for a single effect was carried out using the isobologram. A more general isobolar analysis that examines combinations of compounds over a range of effects would provide more complete information. The occurrence of synergism, antagonism, or additivity depends not only on the compound and the effects measured but also on the fixed ratio combination and the total dose in the combination. A comparison between the theoretical additive curve, obtained from curves of individual compounds for each fixed ratio, and the experimental curve can be performed as was previously described by Fernández et al. ${ }^{18} \mathrm{~A}$ given interaction type (synergism, additivity, or antagonism) at some midrange effect may be extended to other dose levels when the theoretical regression line and the experimental data are compared. On applying Student's $t$ test, a value of $95 \%$ is sufficient to distinguish whether the slopes between lines differ significantly. 18

The changes detected in the type of effect for the different combinations, depending on the dose, were evaluated by analyzing the $\mathrm{ED}_{25}, \mathrm{ED}_{50}$, and $\mathrm{ED}_{75}$ isobolograms. These changes were analyzed using the dose-effect curve by applying Student's $t$ test (95\%) of parallelism for linear models between the additive curve and the experimental curve (Table 2).

For all the combinations tested, binary mixtures with compounds $1 / 3,2 / 4$, and $3 / 4$ in the corresponding ratios, the types of interaction in their isobolograms $\left(\mathrm{ED}_{25}, \mathrm{ED}_{50}\right.$, and $\left.E D_{75}\right)$ remain constant over different ranges of measured effects. Deviations from this behavior were found for the combinations $1 / 2$ in the $1: 3,1: 1$, and $3: 1$ ratios, for $\mathbf{1} / \mathbf{4}$ in the $3: 2$ ratio, and for $3 / 2$ in the $3: 1$ ratio, for which the interactions were not constant for different ranges of measured effects in the isobolograms $\left(\mathrm{ED}_{25}, \mathrm{ED}_{50}\right.$, and $\left.\mathrm{ED}_{75}\right)$ (Tables 1 and 2). The additive and experimental regression lines for these binary mixtures in the aforementioned ratios are shown in Figures 5-9.

The additive and experimental regression lines for the binary mixtures of aguerin B (1)/grosheimin (2) in all ratios (3:1, 1:1, and 1:3) tested in the $\mathrm{ED}_{25}, \mathrm{ED}_{50}$, and $\mathrm{ED}_{75}$ normalized isobolograms showed changes in the type of effect for the three ratios. These effects changed from additive to synergistic. This trend can be observed by comparing the additive and experimental regression lines (Figures 5-7); increases in the 


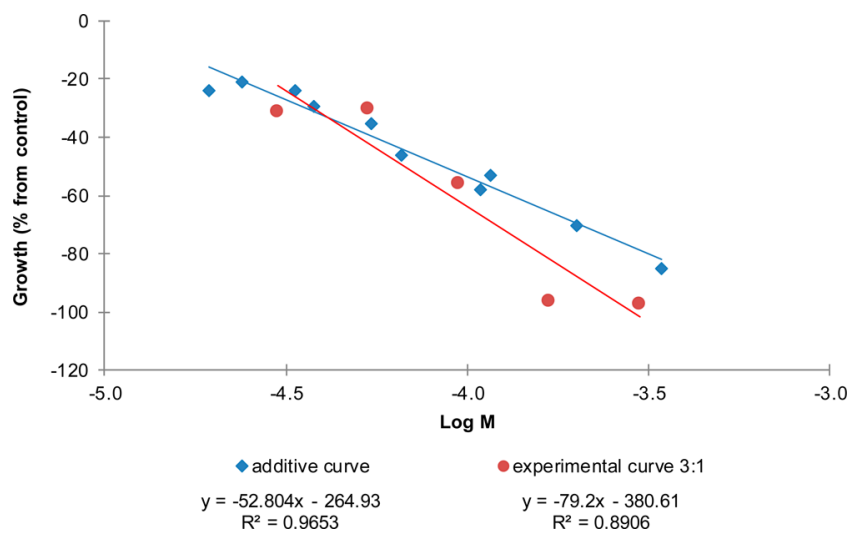

Figure 5. Comparison of the additive and experimental regression lines for the combination of aguerin B (1)/grosheimin (2) in a 3:1 ratio.

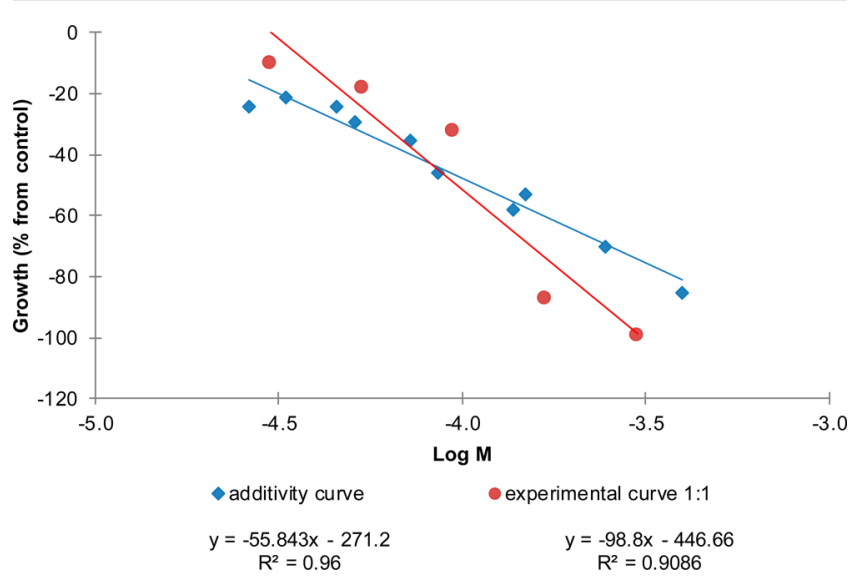

Figure 6. Comparison of the additive and experimental regression lines for the combination of aguerin B (1)/grosheimin (2) in a $1: 1$ ratio.

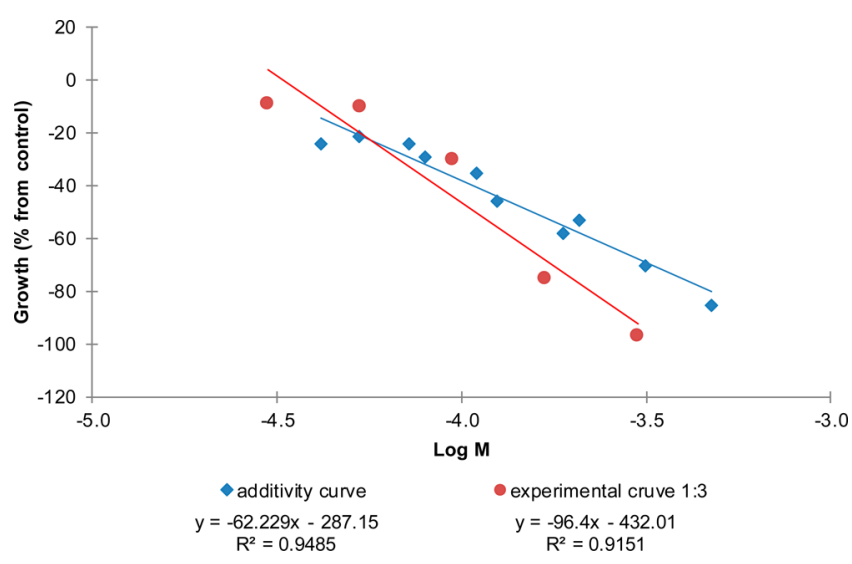

Figure 7. Comparison of the additive and experimental regression lines for the combination of aguerin B (1)/grosheimin (2) in a 1:3 ratio.

doses of the combination shift the experimental curve to lower values with respect to the additive curve for the same effect level-a change that is indicative of synergistic effects (at high doses). These qualitative data graphs, for which Student's $t$ test (95\%) shows significant differences between the slopes of the additive and experimental curves, are consistent with the trend of decreasing interaction index $(\lambda)$ values, which even drop below 1 . The first value is $\lambda=1.02$ at $\mathrm{ED}_{25}$, and this is followed by $\lambda=0.67$ at $\mathrm{ED}_{50}$ and finally $\lambda=0.54$ at $\mathrm{ED}_{75}$. The most prominent synergistic effect was observed at this level by virtue of the lower value for the interaction index $(\lambda=0.54)$ (Table 1).

Similarly, the results for the combination of compounds aguerin B (1)/11,13-dihydroxy-8-desoxygrosheimin (4) in a $3: 2$ ratio showed a change from an additive effect in the $\mathrm{ED}_{25}$ and $\mathrm{ED}_{50}$ isobolograms to a synergistic effect at inhibition level of $75 \%$, as shown by the $\mathrm{ED}_{75}$ isobologram. This fact can be observed by comparing the additive and experimental regression lines (Figure 8), where Student's $t$ test showed a

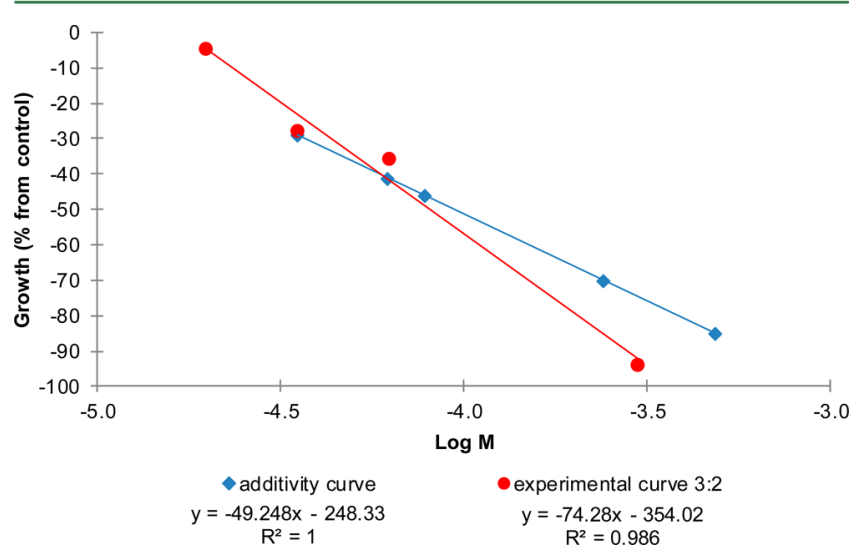

Figure 8. Comparison of the additive and experimental regression lines for the combination of aguerin B (1)/11,13-dihydroxy-8desoxygrosheimin (4) in a 3:2 ratio.

significant difference between the slopes of the linear model for both curves, and this led to a smaller dose for the experimental curve than for the additive curve for levels $>50 \%$ inhibition. This trend is corroborated by the change in the interaction index $(\lambda)$ from 1.14 in the $\mathrm{ED}_{25}$ isobologram to values below unity $(\lambda=0.44)$ in the $\mathrm{ED}_{75}$ isobologram (Table 1$)$.

Finally, the additive and experimental regression lines for the combination of compounds cynaropicrin (3)/grosheimin (2) in a 3:1 ratio are represented in Figure 9. It can be observed that this interaction was not constant for the different ranges of measured effects. This graph shows an antagonistic effect in the $\mathrm{ED}_{25}$ normalized isobologram, but from inhibition values of

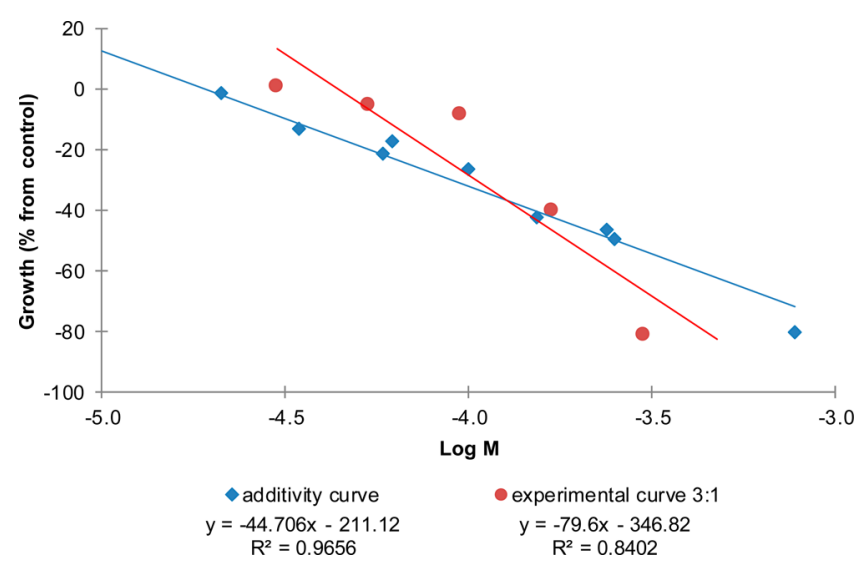

Figure 9. Comparison of the additive and experimental regression lines for the combination of cynaropicrin (3)/grosheimin (2) in a 3:1 ratio. 
$50-75 \%$ this effect becomes synergistic. The trend can be observed by comparing the additive and experimental regression lines (Figure 9), for which Student's $t$ test showed a significant difference (Table 2). For the same effect level, when the doses of the combination $(\geq 50 \%)$ are increased, the experimental curves show a smaller dose than the additive curve and the distance between these lines increases for the higher doses. This situation was corroborated by the lower interaction index for the synergistic effect: 0.35 for $75 \%$ inhibition (Table 1).

In the five cases where there are differences between the theoretical and experimental curves, at higher doses a synergistic effect is observed, and this increases in magnitude with increasing doses. This trend for different binary combinations of compounds with additive and synergistic effects is maintained at high doses (Table 1) and is far more prevalent than cases that show antagonism. These findings may explain to some extent the slight variations observed at higher doses in the activity profiles in wheat coleoptile bioassays, with ethyl acetate extract and with the most bioactive fractions (D, F, G) (Figure 1A,B) when compared to the activity profiles obtained for the pure compounds (Figure 1C). ${ }^{5}$ Compounds 1, 2 , and 3 showed high inhibitory activity on coleoptile elongation at the first two concentrations (at $10^{-3} \mathrm{M}$ with almost $90 \%$ activity and at $3 \times 10^{-4} \mathrm{M}$ with 70,75 , and $55 \%$ inhibition, respectively), and their activities decreased markedly with dilution. However, with the extracts and fractions were retained high activity levels upon dilution. In contrast, compound $\mathbf{4}$ achieved only $20 \%$ inhibition at the highest concentration $\left(10^{-3} \mathrm{M}\right)$.

The trends observed in this study may be due to the following: (a) Interactions in the extract and fractions are not exclusively due to only one or two compounds, and other minor compounds could also be involved; (b) the four sesquiterpene lactones (1-4) are involved in all interaction effects detected, and it should be borne in mind that compound 4 does not have inherent activity in the wheat coleoptile bioassay.

This second hypothesis is supported by the following findings. Compound 4 participates in all three types of interaction; it showed synergism with compound $\mathbf{1}$ at high doses (75\%), additivity with compounds 1 (at lower and middle doses, 25 and 50\%) and 2 (at all doses, 25, 50, and $75 \%$ ), and antagonism with compound 3 at $25 \%$ inhibition. This implies that these interaction effects are present in fraction $\mathrm{G}$, from which compound 4 was isolated. In this way, some authors support a fractionation-biodirected synergistic approach because this is considered to be a more tactical study of the compounds involved in interactions in complex mixtures. ${ }^{30}$ Therefore, the joint action of major sesquiterpene lactones isolated from $C$. cardunculus can explain the activities observed in extracts and fractions.

The results described above emphasize the validity of experiments on binary mixtures using the coleoptile bioassay and its potential application, especially for test systems in initial screenings, before studying such binary mixtures by more specific bioassays. These specific bioassays are required to determine the potential effects of sesquiterpene lactones on other interactions in binary and complex mixtures. Such studies should deepen the understanding of the significant potential of C. cardunculus for use in future biological applications.

\section{AUTHOR INFORMATION}

\section{Corresponding Author}

*(F.A.M.) Phone: +34 956.012.770. Fax: +34 956.016.193. Email: famacias@uca.es.

\section{Funding}

This research was supported by the Ministerio de Economía y Competitividad (MINECO) (Project AGL2013-42238-R) and Consejería de Economía Innovación y Ciencia, Junta de Andalucía (Project DP12-TEP-725).

\section{Notes}

The authors declare no competing financial interest.

\section{REFERENCES}

(1) Kelly, M. Cynara cardunculus. In Invasive Plants of California's Wildlands; Bossard, C. C., Randall, J. M., Hoshovsky, M. C., Eds.; University of California Press: Berkeley, CA, USA, 2000; pp 139-145.

(2) White, V. A.; Holt, J. S. Competition of artichoke thistle (Cynara cardunculus) with native and exotic grassland species. Weed Sci. 2005, $53,826-833$.

(3) Wixted, K. L.; McGraw, J. B. Competitive and allelopathic effects of garlic mustard (Alliparia petiolata) on American ginseng (Panax quinquefolius). Plant Ecol. 2010, 208, 347-357.

(4) Morvillo, C. M. d. l. F.; de la Fuente, E. B.; Martinez-Ghersa, M. A.; Gonzalez-Andujar, J. L. Competitive and allelopathic interfernce between soybean crop and annual wormwood (Artemisia annua L.) under field condition. Eur. J. Agron. 2011, 34, 211-221.

(5) Rial, C.; Novaes, P.; Varela, R. M.; G. Molinillo, J. M.; Macias, F. A. Phytotoxicity of cardoon (Cynara cardunculus) allelochemicals on standard target species and weeds. J. Agric. Food Chem. 2014, 62, 6699-6706.

(6) Huang, Z.; Haig, T.; Wu, H.; An, M.; Pratley, J. Correlation between phytotoxicity on annual ryegrass (Lolium rigidum) and production dynamics of allelochemicals within root exudates of an allelopathic wheat. J. Chem. Ecol. 2003, 29, 2263-2279.

(7) Jia, C.; Kudsk, P.; Mathiassen, S. K. Joint action of benzoxazinone derivatives and phenolic acids. J. Agric. Food Chem. 2006, 54, 10491057.

(8) Einhellig, F. A. Allelopathy: current status and future goals. In Allelopathy, Organisms, Processes and Applications; Inderjit, Dakshini, $\mathrm{K}$. M. M., Einhellig, F. A., Eds.; American Chemical Society: Washington, DC, USA, 1995; pp 1-24.

(9) Einhellig, F. A.; Rasmussen, J. A. Synergistic inhibitory effects of vanillic and $p$-hydroxybenzoic acids on radish and grain sorghum. $J$. Chem. Ecol. 1978, 4, 425-36.

(10) Rasmussen, J. A.; Einhellig, F. A. Inhibitory effects of combinations of three phenolic acids on grain sorghum germination. Plant Sci. Lett. 1979, 14, 69-74.

(11) Inderjit; Streibig, J. C.; Olofsdotter, M. Joint action of phenolic acid mixtures and its significance in allelopathy research. Physiol. Plant. 2002, 114, 422-428.

(12) Greco, W. R.; Bravo, G.; Parsons, J. C. The search for synergy: a critical review from a response surface perspective. Pharmacol. Rev. 1995, 47, 331-385.

(13) Jonker, M. J.; Svendsen, C.; Bedaux, J. J. M.; Bongers, M.; Kammenga, J. E. Significance testing of synergistic/antagonistic, dose level-dependent, or dose ratio-dependent effects in mixture doseresponse analysis. Environ. Toxicol. Chem. 2005, 24, 2701-2713.

(14) Wehtje, G. R.; Wilcut, J. W.; Dylewski, D. P.; McGuire, J. A.; Hicks, T. V. Antagonism of paraquat phytotoxicity in peanuts (Arachis hypogaea) and selected weed species by naptalam. Weed Sci. 1991, 39, 634-639.

(15) Scherder, E. F.; Talbert, R. E.; Lovelace, M. L. Antagonism of cyhalofop grass activity by halosulfuron, triclopyr, and propanil. Weed Technol. 2005, 19, 934-941.

(16) Jonker, M. J.; Sweijen, R.; Kammenga, J. E. Toxicity of simple mixtures to the nematode Caenorhabditis elegans in relation to soil sorption. Environ. Toxicol. Chem. 2004, 23, 480-488. 
(17) Cedergreen, N.; Kudsk, P.; Mathiassen, S. K.; Soerensen, H.; Streibig, J. C. Reproducibility of binary-mixture toxicity studies. Environ. Toxicol. Chem. 2007, 26, 149-156.

(18) Garcia, B. F.; Torres, A.; Macias, F. A. Synergy and other interactions between polymethoxyflavones from citrus by products. Molecules 2015, 20, 20079-20106.

(19) Cutler, H. G. A fresh look at the wheat coleoptile bioassay. In Proceedings of the 11th Annual Meeting of the Plant Growth Regulator Society of America; Cutler, H. G., Ed.; Boston, MA, USA, 1984; pp 19.

(20) Cutler, S. J.; Hoagland, R. E.; Cutler, H. G. Evaluation of selected pharmaceuticals as potencial herbicides: Brindging the gap between agrochemicals and pharmaceuticals. In Allelopathy in Ecological Agriculture and Forestry, Proceedings of the III International Congress; Narwal, S. S.; Hoagland, R. E., Dilday, M. J., Reigosa, M. J., Eds.; Springer: Dordrecht, The Netherlands, 20001998; pp 129-137. (21) Jacyno, J. M.; Cutler, H. G. Detection of herbicidal properties: scope, and limitations of the etiolated wheat coleoptiles biossay. PGRSA Q. 1993, 21, 15-24.

(22) Hancock, C. R.; Barlow, H. W. B.; Lacey, H. J. The behavior of phloridzin in the coleoptile straightgrowth test. J. Exp. Bot. 1961, 12, 401-408.

(23) Nitsch, J. P.; Nitsch, C. Growth of coleoptile and first internode sections. A new, sensitive, straight-growth test for auxin. Plant Physiol. 1956, 31, 94-111.

(24) Macias, F. A.; Simonet, A. M.; Pacheco, P. C.; Barrero, A. F.; Cabrera, E.; Jimenez-Gonzalez, D. Natural and synthetic podolactones with potential use as natural herbicide models. J. Agric. Food Chem. 2000, 48, 3003-3007.

(25) Martín Andrés, A.; Luna del Castillo, J. D. Bioestadistica para las Ciencias de la Salud; Ediciones Norma: Madrid, Spain, 1990; pp 1722.

(26) Tallarida, R. J. Drug Synergism and Dose-Effect Data Analysis, 1st ed.; Chapman \& Hall/CRC Press: Boca Raton, FL, USA, 2000; pp 1247.

(27) Tallarida, R. J. The interaction index: a measure of drug synergism. Pain 2002, 98, 163-168.

(28) Tallarida, R. J. Drug synergism: its detection and applications. J. Pharmacol. Exp. Ther. 2001, 298, 865-872.

(29) Berenbaum, M. C. What is synergy? Pharmacol. Rev. 1989, 41, 93-141.

(30) Junio, H. A.; Sy-Cordero, A. A.; Ettefagh, K. A.; Burns, J. T.; Micko, K. T.; Graf, T. N.; Richter, S. J.; Cannon, R. E.; Oberlies, N. H.; Cech, N. B. Synergy-directed fractionation of botanical medicines: a case study with goldenseal (Hydrastis canadensis). J. Nat. Prod. 2011, 74, 1621-1629.

(31) Caccioni, D. R. L.; Guizzardi, M.; Biondi, D. M.; Renda, A.; Ruberto, G. Relationship between volatile components of citrus fruit essential oils and antimicrobial action on Penicillium digitatum and Penicillium italicum. Int. J. Food Microbiol. 1998, 43, 73-79. 